\section{Estado de la implementación del Sistema de Seguridad y Salud en el Trabajo en el sector lácteo en La Calera Cundinamarca}

\section{Status of the implementation of the Occupational Health and Safety System in the dairy sector of the municipality of La Calera Cundinamarca}

\section{Edilma González Buitrago}

Administradora en Salud Ocupacional, Tecnóloga en Gestión de Mercados, miembro del Semillero de Investigación SEISS, Corporación Universitaria Minuto de Dios, Inspector HSE en Equipos \& Cimentaciones, egonzalezb3@uniminuto.edu.co, ORCID ID https://orcid.org/oooo-0oo3-1778-049.

\section{Héctor Peña Chacón}

Administrador en Salud Ocupacional, Ingeniero Civil, Especialista en Construcción Sostenible, miembro del Semillero de Investigación SEISS, Corporación Universitaria Minuto de Dios, Tecnólogo operativo en Dirección gestión ambiental del sistema hídrico de la Empresa de Acueducto de Bogotá, ORCID https://orcid.org/o0oo-00027418-5613, hpenachacon@uniminuto.edu.co

\section{Sinndy Dayana Rico Lugo .}

Ingeniera Industrial, Magíster en Ingeniería de Producción, Especialista en Ingeniería de

Producción y Logística, sinndydayana.rico@gmail.com, ORCID ID https://orcid.org/oooo-0002-68635139 .

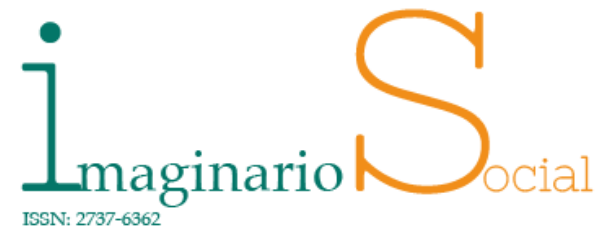

Imaginario Social

e-ISSN: $2737-6362$

enero - junio 2020 Vol. 3-1-2020

http://revista-

imaginariosocial.com/index.php/es/in dex

Recepción: 23 de abril 2019

Aceptación: 15 de octubre 2019

$32-48$

Atribución/Reconocimiento-NoCo mercial- Compartirlgual 4.0 Licencia Pública Internacional - CC BY-NC-SA 4.0

https://creativecommons.org/licenses /by-nc- sa/4.0/legalcode.es 


\title{
Resumen
}

El estudio se desarrolló en 2018 para conocer la gestión organizacional respecto al Sistema de Gestión de Seguridad y Salud en el Trabajo de las empresas legalmente constituidas del sector lácteo del municipio de La Calera-Cundinamarca. Se identificaron las empresas a partir de diversas bases de datos, procediendo a realizar observación en campo y entrevistas a sus representantes legales y a funcionarios de entidades oficiales. La investigación determinó que los microempresarios no tienen la capacidad para implementar dicho sistema.

Palabras clave: Sistema de gestión, seguridad y salud en el trabajo, sector lácteo

\begin{abstract}
The study was developed in 2018 to know the organizational management regarding the Occupational Health and Safety Management System of the legally constituted companies of the dairy sector of the municipality of La Calera-Cundinamarca. Companies were identified from various databases, proceeding to conduct field observation and interviews with their legal representatives and officials of official entities. The investigation determined that microentrepreneurs do not have the capacity to implement such a system.
\end{abstract}

Key word: Management system, safety and health at work, dairy sector.

\section{Introducción}

Colombia, a pesar de ser un país aún en desarrollo cuya principal fuente económica son sus recursos naturales, ha tenido en cuenta la prevención de accidentes y enfermedades laborales garantizando procesos seguros y controlados donde el trabajador goce de bienestar físico y mental y las pérdidas por daños de equipos, maquinaria, etc, sean minimizados posibilitando que la economía del país pueda ser sostenible, sustentable y competente para el mundo. Así, la reglamentación del sector trabajo lucha por establecer procesos seguros en las empresas, lo que conlleva a establecer los Estándares Mínimos del Sistema de Gestión de la Seguridad y Salud en el Trabajo establecidos en la Resolución 1111 de 2017 (Ministerio de Trabajo, 2017).

Si bien la obligatoriedad de Ley establece el porcentaje que las empresas deben cumplir respecto a la implementación del SG - SST, se debe tener en cuenta que el (...) Sistema de Gestión de la Seguridad y la Salud en el Trabajo -SG-SST es un proceso 
lógico y por etapas, basado en la mejora continua cuyo objetivo es reconocer, evaluar y controlar los riesgos que puedan afectar la empresa (Social M. d., 2012). Esto implica que las empresas deben estar capacitadas para implementar de manera efectiva este sistema de gestión, lo que es una limitante en varios municipios agropecuarios del país.

Dentro de ese tipo de municipios se encuentra La Calera Cundinamarca, que tiene una vocación de carácter agropecuario poco tecnificado principalmente gracias a la riqueza de productos naturales y a la ausencia de formación en gran parte de la población que en su mayoría es campesina como lo indica el Plan de Desarrollo del municipio de La Calera Cundinamarca en el (...) (Vargas, 2016). Dichas características se han consolidado como un obstáculo para llevar a cabo ese proceso lógico y sistemático asociado a la implementación del SG-SST que exige formación en gestión de sistemas empresariales.

\subsection{Finalidad de estudio}

La investigación se enfocó en diagnosticar el estado del arte de la implementación del Sistema de Gestión de la Seguridad y la Salud en el Trabajo de las empresas legalmente constituidas del municipio de La Calera Cundinamarca cuya actividad económica correspondiera al sector lácteo; con el fin de establecer el cumplimiento de la normatividad vigente dispuesto en el Decreto 1072 de 2015, Capitulo 6, Titulo 4, Parte 2, l; "que las empresas legalmente constituidas están obligadas a implementar el SGSST (Trabajo, 2015).

Dicho lo anterior se establece la necesidad de conocer ¿Cuál es el estado de implementación del Sistema de Gestión de Seguridad y Salud en el Trabajo en empresas del sector lácteo del municipio de La Calera Cundinamarca?

\subsection{Importancia del SG-SST en la población objeto de estudio}

Esta investigación es producto de la participación de los autores en el Semillero de Innovación Sostenible y Seguridad "SEISS" que promueve la investigación de los estudiantes del centro tutorial Guasca, incluyendo La Calera y Gacheta, y busca concientizar a los participantes entorno a la necesidad y utilidad de los proyectos de innovación como un aporte relevante al crecimiento económico de la provincia del Guavio, pertenece a la regional Zipaquirá de la Corporación Universitaria Minuto de 
Dios y los proyectos allí desarrollados se diseñan a partir del refinamiento de ideas que pretenden identificar y plantear soluciones a problemáticas de la región de residencia de los participantes con un enfoque de innovación y emprendimiento teniendo en cuenta dos claros componentes; el desarrollo sostenible y la salud y seguridad en el trabajo;

Los autores guiados por la idea de conocer el estado de implementación del Sistema de Gestión de la Seguridad y la Salud en el Trabajo de las empresas del sector lácteo legalmente constituidas del municipio de La Calera Cundinamarca se enfocan en la línea de investigación de competitividad y desarrollo económico y en la sub-línea de investigación seguridad y salud en el trabajo en poblaciones de alto impacto y de interés especial.

En un estudio inicial aplicado en general al Municipio de La Calera Cundinamarca se conoció que el estado actual de implementación del Sistema de Gestión de la Seguridad y Salud en el Trabajo SG-SST en las empresas legalmente constituidas, en general no responde al ideal de la norma vigente puesto que se estipulan reglas en aras de prevenir accidentes y enfermedades laborales garantizando bienestar en las diferentes áreas de trabajo; entonces estas empresas expresamente cumplen lo mínimo y en la parte documental, lo hacen por evitar sanciones y garantizar su funcionamiento; de acuerdo a este concepto se aprecia que no intervienen desde una perspectiva propia de cultura de prevención.

En un estudio inicial aplicado en general al Municipio de La Calera Cundinamarca se conoció que el estado actual de implementación del Sistema de Gestión de la Seguridad y Salud en el Trabajo SG-SST en las empresas legalmente constituidas en general no responde al ideal de la norma vigente, puesto que se estipulan reglas en aras de prevenir accidentes y enfermedades laborales garantizando bienestar en las diferentes áreas de trabajo; entonces estas empresas expresamente cumplen lo mínimo y en la parte documental, lo hacen por evitar sanciones y garantizar su funcionamiento; de acuerdo a este concepto se aprecia que no intervienen desde una perspectiva propia de cultura de prevención. 
El proyecto de investigación es llevado a cabo dentro del territorio del municipio de La Calera Cundinamarca ubicado al occidente de la provincia del Guavio, y al nororiente de Bogotá, hace parte de los municipios de la sabana de Bogotá, ha sido un municipio Colono dedicado a la agricultura y la explotación de piedra caliza desde su fundación hace 246 años;(...) (Departamento Nacional de Planeación, DNP, 2014 - 2015)La Calera Cundinamarca cuenta con aproximadamente 27527 habitantes, y una geografía montañosa y rocosa en su gran mayoría, así como su clima frio.

Como ya se ha dicho en el plan de desarrollo del municipio de La Calera de su vocación agropecuaria y con una amplia población de tradición campesina que requiere unos nuevos enfoques orientados al ordenamiento del territorio la protección del medio ambiente, el desarrollo sostenible y crecimiento económico positivo y de alto impacto que garantice el bienestar de las futuras generaciones (Vargas, 2016). Por esta razón, los autores deciden encausar la investigación en las empresas del sector lácteo del municipio.

Durante el desarrollo investigativo se logró identificar que en el municipio de La Calera Cundinamarca pequeños ganaderos no han logrado constituir legalmente su empresa lo que les convierte en pequeños comerciantes informales manipulables de quienes tienen el grueso del negocio como lo son Alquería, Alpina, quienes atesoran gran porcentaje de la producción lechera de la región, imponiendo sus reglas de juego como por ejemplo el precio.

\subsection{Estado del arte}

Como se mencionó, (...) el Plan Nacional de Seguridad y Salud en el Trabajo (PNSST) se piensa como instrumento de gestión integral, de esta forma otorga relevancia a las necesidades que actualmente tiene Colombia respecto del área de salud laboral (Min, s.f.), tal y como se estableció en la Ley 1562 de 2012 que trae consigo la transformación de un Sistema de Riesgos Laborales más justo, equitativo, y eficiente. (Colombia C. d., 2012)

Este Plan Nacional se formuló con la participación del Comité Nacional de Seguridad y Salud en el Trabajo, la academia, centros de investigación, las administradoras de Riesgos Laborales, empresas promotoras de la salud, instituciones prestadoras de 
servicios de Salud Ocupacional (hoy Seguridad y Salud en el Trabajo), entidades gubernamentales, agremiaciones, empleadores, organizaciones sindicales $\mathrm{y}$ trabajadores.

Para el año 2014 se expidió la Resolución 6045 por la cual se adoptó el Plan Nacional de Seguridad y Salud en el Trabajo 2013-2021. (trabajo M. d., Resolución 6045, 2014) En la implementación de este Plan Nacional se contemplaron cuatro estrategias a saber:

- $\quad$ Fomento de la transversalidad de la SST en el conjunto de políticas públicas.

- $\quad$ Fortalecimiento institucional de la Seguridad y la Salud en el Trabajo.

- $\quad$ Fortalecimiento de la promoción de la seguridad, de la salud de los trabajadores y la prevención de los riesgos laborales.

- Optimización y garantía del reconocimiento de las prestaciones al trabajador en el sistema general de riesgos laborales.

Algunos de los resultados de la evaluación del Plan Nacional de Seguridad y Salud en el Trabajo 2013-2021 son:

- El cumplimiento global de todos los actores fue del 60\%, se observa un incremento de 10 puntos con respecto al año 2015.

- En relación con la expedición y actualización de normas en materia de seguridad y salud en el trabajo, se observa un avance del $77 \%$, identificando un incremento de 19 puntos con respecto al 2015, enfocando al fomento del seguimiento y cumplimiento de normas nacionales y de convenios internacionales, fortaleciendo la protección de la SST de los trabajadores.

- Se evidencia un aumento en el desarrollo de campañas y estrategias de divulgación, educación, capacitación, sensibilización (videos, afiches, cartillas, cuñas radiales, etc.) dirigidas a promover la salud y prevenir accidentes de trabajo y enfermedades laborales a empresas, trabajadores y demás actores del Sistema General de Riesgos Laborales, incrementándose en 5 puntos con respecto al año anterior y logrando un $75 \%$ de avance. 
- $\quad$ Se logró un 71\% de avance en la elaboración de guías y manuales para la implementación del SG SST, las cuales contienen herramientas, formatos, instrumentos y señalan paso a paso como desarrollarlo, avanzando en 10 puntos frente al 2015 .

- $\quad$ Fortalecimiento de los Comités Seccionales y Locales de Seguridad y Salud en el Trabajo. Se realizaron visitas de asistencia técnica en SST y acompañamiento a los comités seccionales y locales del SST en 2015, 2016 y 2017, incrementándose en 15 puntos porcentuales la participación de estos en la formulación de planes de acción armonizados con el Plan Nacional de Seguridad y Salud en el Trabajo (Trabajo M. d., 2017)

Una vez consultadas las diferentes bases de datos a las que se tuvo acceso, no fue posible identificar alguna fuente de información que puntualice el estado de avance de implementación del SG-SST en el municipio de La Calera Cundinamarca; por lo tanto, no se halló información específica en el sector lácteo.

\subsection{Teorías asociadas}

Desde inicio del siglo XX se adoptaron leyes y normas para facilitar la implementación de la salud ocupacional que se concentraron principalmente en minería, construcción, agricultura y otros procesos de alto riesgo donde los índices de accidentalidad y enfermedad se incrementaron por falta de control en los procesos operativos de las organizaciones. Con la Ley 9 de 1979 (Colombia, 1979) se dictan medidas sanitarias, y objeta el tema de Salud Ocupacional, implementa plan de emergencias, vigilancia y control, entre otros, afirma también la Resolución 2013 de 1986 (Social M. d., 1986) para la conformación de un Comité Paritario de Salud Ocupacional (COPASO) en las empresas, luego crea la Ley 361 DE 1997 (República, 1997)estableciendo mecanismos de integración social de las personas con limitación y se dictan otras disposiciones.

Pasadas casi dos décadas las organizaciones continuaban inmersas en la informalización y el afán por las utilidades a bajo costo; entonces fue estipulada la Ley 378 DE 1997 (Congreso de La República , 1997)Por medio de la cual se aprueba el "Convenio número 161, sobre los servicios de salud en el trabajo"; años más tarde interviene con la Ley 776 DE 2002 (Congreso de Colombia, 2002)Por la cual se dictan 
normas sobre la organización, administración y prestaciones del Sistema General de Riesgos Profesionales, y refuerza con la Ley 1010 DE 2006 (Colombia, 2006) donde se adoptan medidas para prevenir, corregir y sancionar el acoso laboral y otros hostigamientos en el marco de las relaciones de trabajo.

Para ese entonces solo un pequeño porcentaje de empresas aplicaba la normatividad en Salud Ocupacional; entonces el Ministerio del Trabajo en el año 2012 inicia con la obligatoriedad de Ley en las siguientes condiciones de norma:

Ley 1562 DE 2012 (Social M. d., 2012) donde se modifica el Sistema de Riesgos Laborales y se dictan otras disposiciones en materia de Salud Ocupacional; en el Decreto 1443 de 2014 (Trabajo, 2014)dicta disposiciones para la implementación del Sistema de Gestión de la Seguridad y Salud en el Trabajo (SG-SST), no obstante el país continuaba en crisis económica y se presentaban alteraciones de orden público como el paro agrario sucedido en los años 2013-2014, lo que obligó a establecer garantías laborales, y publica el Decreto Número 1072 de 2015 (Trabajo, 2015). Compilando la Reglamentación del Sector Trabajo, la lucha por establecer procesos seguros en las empresas conlleva a establecer los Estándares Mínimos del Sistema de Gestión de la Seguridad y Salud en el Trabajo, y los dicta bajo la Resolución 1111 de 2017 (Ministerio de Trabajo, 2017).

\section{Materiales y Métodos}

La investigación tuvo enfoque mixto, que comenzó con carácter cualitativo con el objetivo de describir un fenómeno basado en datos ya existentes como es la implementación del SG-SST, y luego durante el proceso se abonaron características cuantitativas basadas en estadísticas que aportaron al análisis de la realidad objetiva asociada al número de empresas legalmente constituidas y su estado de implementación en relación con el SG-SST. En este contexto, el alcance de la investigación fue inicialmente exploratorio puesto que el estado de avance del SG-SST en el municipio era desconocido. Posteriormente, con la caracterización de las empresas se pasó a la etapa descriptiva. Así, las técnicas e instrumentos de recolección de información fueron revisión documental durante la etapa exploratoria, y encuestas 
y entrevistas para la etapa descriptiva. En la Figura 1 se presenta el flujo de desarrollo de la metodología utilizada por esta investigación.

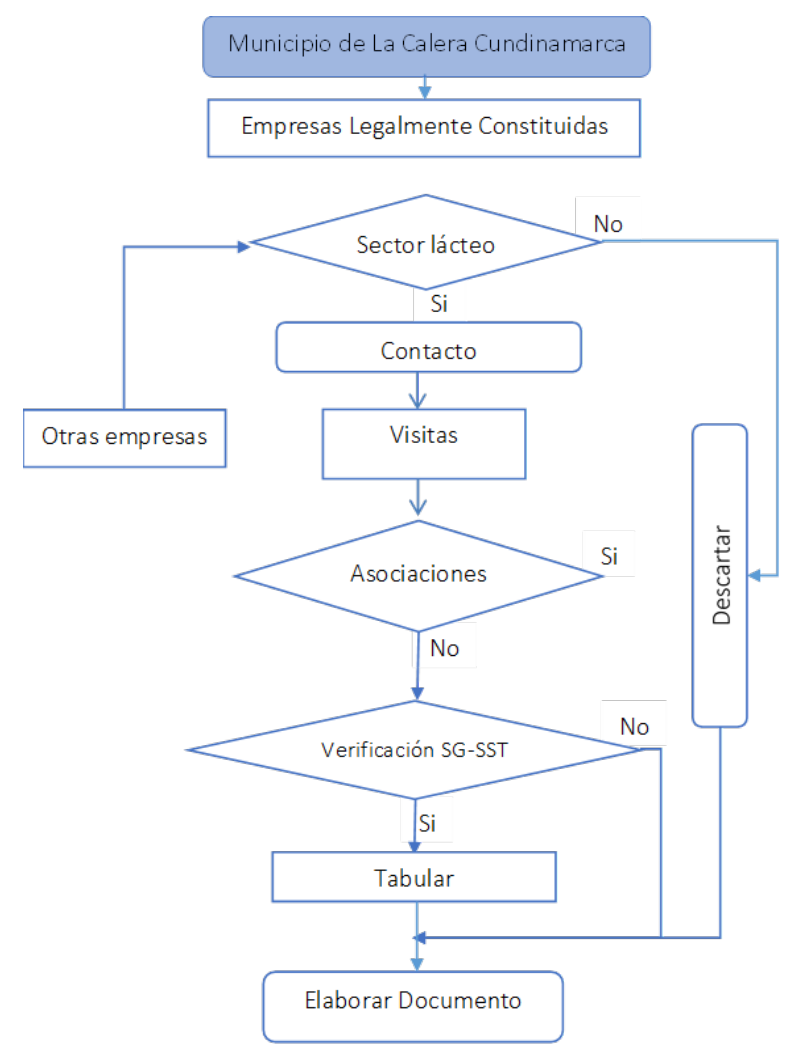

Figura 1. Diagrama de flujo metodológico.

Para la definición de la población a la que se aplicaría el diagnóstico que para la fecha otorgaba la resolución 1111 de 2017 (Ministerio de Trabajo, 2017), se determinaron los siguientes lineamientos progresivos para su caracterización: que estuvieran legalmente constituidas, que tuvieran sede en el municipio de La Calera y que pertenecieran al sector lácteo. A partir de ello se procedió a contactarlas y formalizar visitas en las cuales se verificó el estado de implementación del SG-SST que luego fue tabulado como estadísticas de estado de implementación. Así, la población fue analizada en su totalidad.

\section{Resultados}

\section{Fase 1: Consulta de datos}


En la Tabla 1 se presentan las bases de datos de acceso libre consultadas para identificar las empresas legalmente constituidas del sector lácteo en el municipio.

Tabla 1. Fuentes primarias consultadas

\begin{tabular}{|c|c|}
\hline NOMBRE & CONTENIDO \\
\hline $\begin{array}{l}\text { Cámara de Comercio de } \\
\text { http://www.ccb.org.co/ }\end{array}$ & $\begin{array}{l}\text { Información de las } \\
\text { empresas registradas en } \\
\text { el país por } \\
\text { comúgimen } \\
\text { simplificado. }\end{array}$ \\
\hline $\begin{array}{l}\text { Guía de Empresas } \quad \text { Colombianas } \\
\text { http://empresas. portafolio.co/localidad/LA- } \\
\text { CALERA/ }\end{array}$ & $\begin{array}{l}\text { Ranking de empresas de } \\
\text { La calera por ventas. }\end{array}$ \\
\hline $\begin{array}{l}\text { Universia.net } \\
\text { https://guiaempresas.universia.net.co/localidad/LA- } \\
\text { CALERA/ }\end{array}$ & $\begin{array}{l}\text { Listado de empresas en } \\
\text { La Calera; Se filtró por } \\
\text { actividad económica. }\end{array}$ \\
\hline
\end{tabular}

Fase 2: Datos encontrado A partir de la revisión se encontró un total de 281 empresas bajo el régimen común y 475 en el régimen simplificado, para un total de 756 organizaciones. De ellas, solo 7 pertenecían al sector lácteo. Ver Figura 3 y Figura 2.

FIGURA 2. EMPRESAS POR RÉGIMEN.

municipio de La Calera Cundinamarca

— Régimen Común Régimen Simplificado
FIGURA 3. EMPRESAS POR SECTOR.

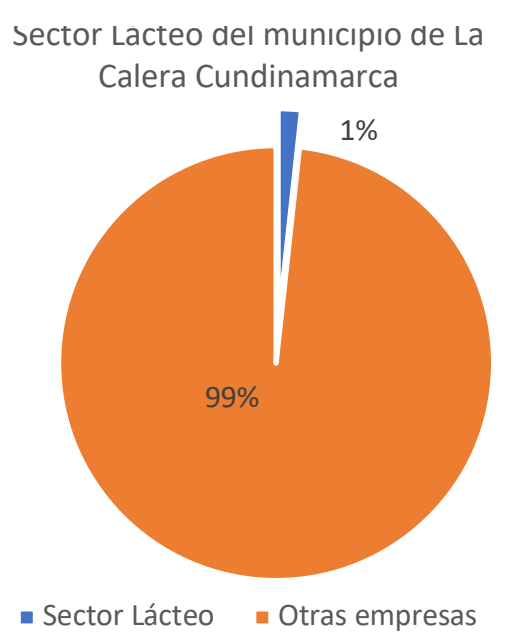




\section{Fase 3: Acercamiento}

En las siguientes gráficas se presentan los resultados respecto a cantidad de empresas que permitieron el agendamiento de visitas para conocer la investigación y revisar la posibilidad de realizar la evaluación del estado de implementación del SG-SST.

Figura 5. RESULtAdos POR ESTRATEGIA DE CONTACTO

FIgURA 4. RESULTADOS ACERCAMIENTO TELEFÓNICO
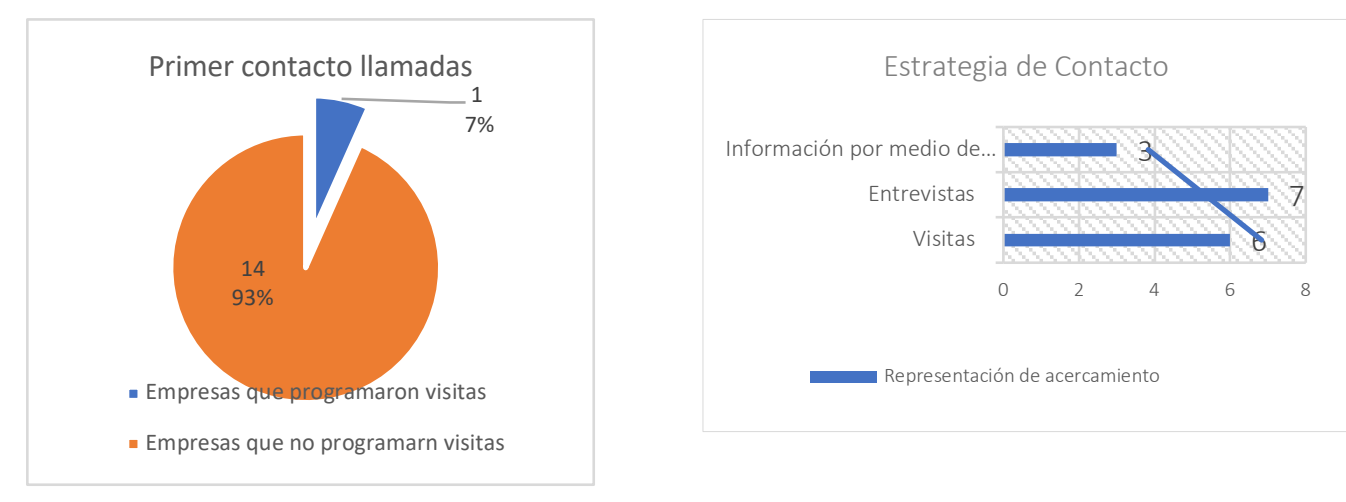

\section{Fase 4: Aplicación Estándares Mínimos}

$\mathrm{Al}$ aplicar el diagnóstico del estado de la implementación del SG-SST de acuerdo con la Resolución 1111 de 2017 vigente a la fecha del estudio, se encontraron los resultados mostrados en la iError! No se encuentra el origen de la referencia., iError! No se encuentra el origen de la referencia., iError! No se encuentra el origen de la referencia., iError! No se encuentra el origen de la referencia. y Figura 10 
Fiqura 7. Resultado estándares Planear

\section{Ciclo I Planear}

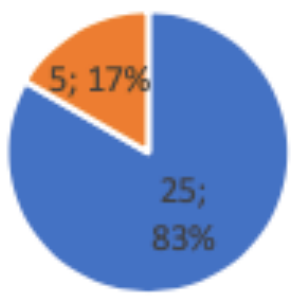

- Puntaje Resolución - Puntaje Obtenido

Fiqura 9. Resultado estándares Verificar Ciclo III Verificar

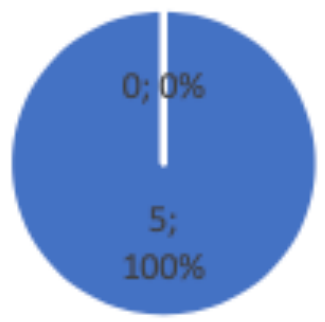

- Purtaje Resdución = Puntaje Obtenido

Figura 10. Resultado diagnóstico final

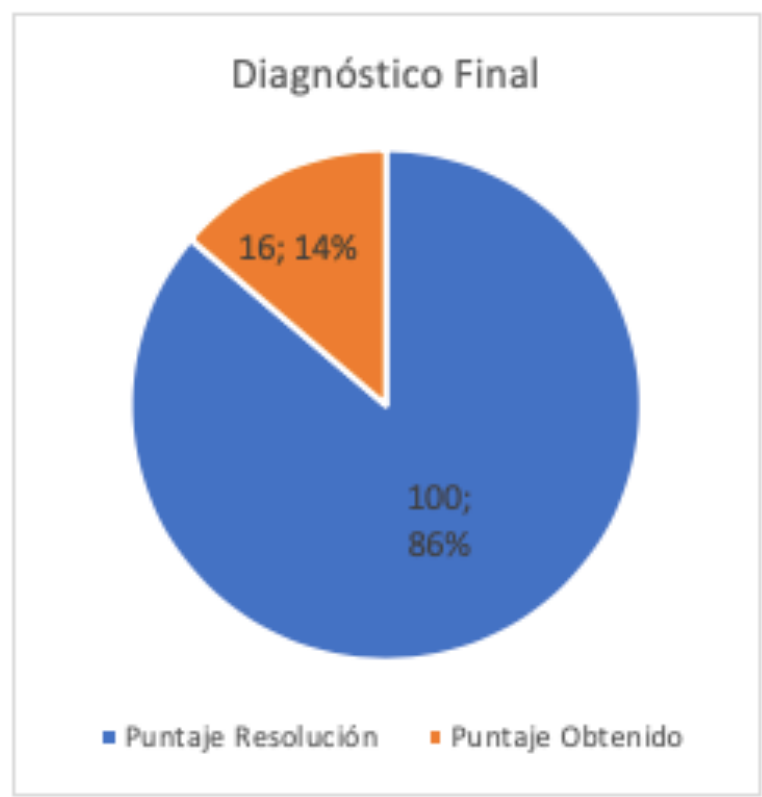

Fiqura 6. Resultado estándares Hacer

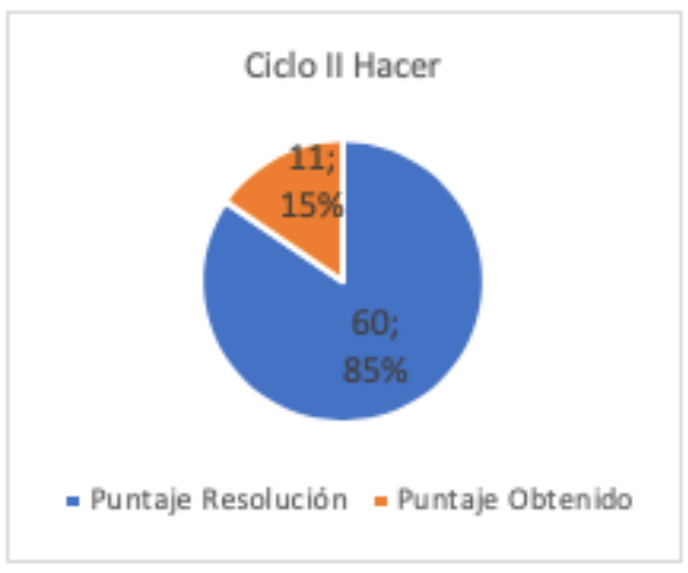

Fiqura 8. Resultado estándares Actuar

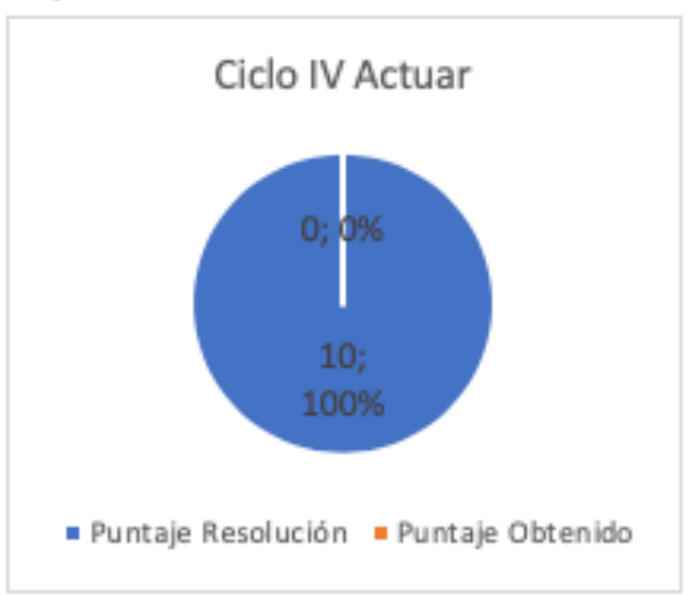




\section{Discusión}

El estado del arte de la implementación del Sistema de Gestión de la Seguridad y la Salud en el Trabajo SG-SST de las empresas legalmente constituidas del municipio de La Calera Cundinamarca y pertenecientes al sector lácteo es un tema que a la fecha de los resultados de la investigación es literalmente desconocido, y obedece a la cultura tradicional del entorno, la misma que genera rechazo al cambio sistemático en el que se encuentra el país por el dinamismo económico que busca competitividad global y brindar garantías a su fuerza laboral.

La población campesina asume este sistema como adverso argumentando atentar contra sus valiosas costumbres y carecer de beneficios de estabilidad ni crecimiento alguno, afirman que el estado genera leyes para favorecer a los grandes empresarios y terratenientes, no para ellos.

De acuerdo con esta percepción los autores definen:

Que aun en la actualidad no hay seguimiento, control ni asesoría idónea de parte de las entidades encargadas; evidenciando que gran parte de este segmento empresarial son personas analfabetas, o con formación mínima y se encuentran en edad avanzada por lo que estos temas resultan densos, inaceptables e incomprensibles para muchos de ellos.

La información de las bases de datos existentes en su mayoría no es completa y no se encuentra actualizada; por esta razón la localización de las empresas enlistadas a realizar es incierta puesto que hacen referencia a domicilios de familias y direcciones inexistentes en la mayoría de casos. Así mismo, al realizar el análisis estadístico de la información, se observa similitud en el 90\% de los casos, una vez verificadas las diferentes bases de datos.

De igual forma, el reconocimiento de la seguridad y la salud en el trabajo es un tema que genera disociación por el desconocimiento de la norma y el tabú existente que otros profesionales han dejado en la historia de implementación del SG-SST 
Por tanto, es evidente en el desarrollo de la investigación la poca importancia y los pocos recursos que asignan los empresarios del municipio de la Calera Cundinamarca a mejorar sus procesos de SG-SST, en especial de las pequeñas y medianas empresas que se resisten a fortalecer el equipo profesional encargado de la implementación del SG-SST, desatendiendo la normatividad al respecto, es esta una de las razones del poco avance que se encuentra en el cumplimiento de la normatividad vigente.

En las empresas investigadas se presenta informalidad en la vinculación laboral respecto a la mano de obra utilizada en los diferentes campos del sector; en cuanto al estado de avance de implementación del SG-SST en las empresas del sector lácteo del municipio de La Calera Cundinamarca legalmente constituidas se halló:

De la totalidad de las empresas filtradas, solamente una empresa se encuentra legalmente constituida y funcionando actualmente; las demás empresas son asociaciones campesinas de productos agrícolas en general cuya producción individual es reducida y se les hace necesario ampliarla, para ello se asocian, además de requerir figura jurídica para comercializar los productos.

No se desarrolla el proceso de sistema de gestión de la seguridad y la salud en el trabajo y las razones más comunes referentes al tema son:

1. Desconocimiento de la norma y de los deberes como persona natural o jurídica cuya actividad económica corresponde al entorno comercial e industrial del sector lácteo

2. Pocos recursos para implementarlo

3. Se aduce ausencia del apoyo gubernamental

Producto de la investigación realizada, en el municipio de La Calera Cundinamarca las empresas legalmente constituidas son pocas; lo que deja en evidencia que en gran porcentaje del sector predomina la informalidad; siendo el municipio de La Calera Cundinamarca agrícola y ganadero, son pocas las personas que le apuestan al sector de transformación de productos lácteos, les ofrece más rentabilidad el vender la leche a grandes empresarios, para lo cual conforman asociaciones como ya se describió en la investigación. 
Se visualiza un amplio panorama de acción, en asesoría de pequeñas y medianas empresas conscientes de su responsabilidad como empleadores y de las posibles sanciones a las que se pueden exponer ya que no han sido abordadas adecuadamente, sea por temas económicos o porque no han sido contactados.

Se determinó que en la actualidad el estado del arte de las empresas legalmente constituidas del municipio de La Calera Cundinamarca enmarcadas en el sector lácteo no se encuentra implementado. Resultado del proceso, solo una de las empresas se encontraba legalmente constituida, pero con un porcentaje de diseño e implementación del SG-SST crítico.

Se argumenta que bajo la actual resolución 0312 que implementa los nuevos estándares mínimos de implementación del Sistema de Gestión de Seguridad y Salud en el Trabajo SG-SST, simplemente se busca otorgar más espacio a la implementación de los mentados Sistemas de Gestión, porque el país en pro de su desarrollo económico no tiene preparado un escenario práctico para la ejecución de las normas en SST, lo que muchas veces deja sin pruebas a las sanciones que en ellas se ajustan.Establecer nuevos estudios y preguntas de investigación.

\section{Conclusiones}

Mediante la investigación se logró observar el estado de avance de implementación del Sistema de Gestión de la Seguridad y la Salud en el Trabajo SG-SST de las empresas legalmente constituidas del municipio de La Calera pertenecientes al sector lácteo y no es acorde a las exigencias que la norma imparte; que los tiempos de ejecución no corresponden a lo estipulado, y que su estado es crítico porque no existe en la mayoría de las empresas.

Así mismo, se identificaron y describieron las empresas del sector lácteo legalmente constituidas en el municipio de La Calera, encontrándose solo una existente y registrada en el régimen común de la Cámara y Comercio. Las demás empresas están registradas como asociaciones de campesinos que en su mayoría no cotizan pensión puesto que el sistema los rechaza por la edad y además no quieren perder el beneficio del SISBEN. 
La aplicación del formato de estándares mínimos de obligatorio cumplimiento acorde a lo establecido en la resolución 1111 de 2017 pudo aplicarse a la única empresa identificada "Lácteos La Veguita”, Obteniendo un puntaje de 16 puntos, entre 1 y 100. A través de la elaboración del informe de resultados donde se describió y tabuló el estado de avance de implementación del SG-SST, así como los demás hallazgos.

El análisis de la información encontrada, producto de la investigación enmarcada en el sector lácteo del municipio llevó a definir que gran parte de la economía local está basada en la informalidad que obedece principalmente al desconocimiento de obligatoriedad de Ley, y la cultura económica del entorno.

Se concluye que la implementación del estado del arte del Sistema de Gestión de Seguridad y Salud en el Trabajo en las empresas del sector lácteo del municipio de La Calera Cundinamarca es muy baja. Sin embargo con la Resolución 0312 de 2019 (Trabajo, 2019), la cual amplía el plazo de transición del SG-SST, define nuevos Estándares Mínimos y deroga la resolución 1111 de 2017, los pequeños empresarios del sector agrícola (incluido el sector lácteos), pueden contar con el apoyo y asesoría de diferentes entidades, gremios o asociaciones para el diseño e implementación del SGSST, lo cual también es una oportunidad para las instituciones de implementar educación por medio de capacitaciones como es el caso de la corporación universitaria minuto de Dios en su programa de Administración en Seguridad y Salud en el Trabajo.Se redacta un número de conclusiones

\section{Referencias}

Colombia, C. $\quad$ d. $\quad$ (1979, $01 \quad$ 24). Obtenido de http://copaso.upbbga.edu.co/legislacion/ley_9_1979.Codigo\%20Sanitario\%20Nacion al.pdf
Colombia,
C.
d. $\quad(2006$,
$0123)$.
Obtenido
de file://C:/Users/User1/Downloads/ley1010230106.pdf

Congreso de Colombia. (2002, $12 \quad$ 17). Obtenido de http://www.secretariasenado.gov.co/senado/basedoc/ley_0776_2002.html
Congres
La República.
(1997,
07
09).
Obtenido
de http://www.secretariasenado.gov.co/senado/basedoc/ley_0378_1997.html 
República,

C.

d.

(1997,

02

07).

Obtenido

de

http://www.secretariasenado.gov.co/senado/basedoc/ley_0361_1997.html

SAMPIERI, R. H. (2010). METODOLOGÍA DE LA INVESTIGACIÓN (Quinta Edición ed.). México D.F: Mc Graw Hill.

Social, M. $\quad$ d. $\quad$ (1986, $06 \quad$ 06). Obtenido de https://www.icbf.gov.co/cargues/avance/docs/resolucion_minsalud_r2013_86.htm

Social, M. d. (2012, 07 11). Ministerio del Trabajo. Obtenido de https://www.minsalud.gov.co/sites/rid/Lists/BibliotecaDigital/RIDE/DE/DIJ/Ley1562-de-2012.pdf

Trabajo, M. $\quad$ d. $\quad$ (2014, $07 \quad 31)$ Obtenido de http://www.mintrabajo.gov.co/documents/20147/36482/decreto_1443_sgsss.pdf/ac 41ab70-e369-9990-c6f4-1774e8d9a5fa

Trabajo,

M. d. (2015, $0526)$.

Obtenido

de

http://www.mintrabajo.gov.co/documents/20147/50711/DUR+Sector+Trabajo+Actua lizado+a+Abril+de+2017.pdf/1f52e341-4def-

8http://www.mintrabajo.gov.co/documents/20147/50711/DUR+Sector+Trabajo+Act ualizado+a+Abril+de+2017.pdf/1f52e341-4def-8d9c-1bee-6e693df5f2d9

Trabajo, M. $\quad$ d. $\quad$ (2017, $03 \quad$ 27). Obtenido de https://www.anm.gov.co/sites/default/files/resolucion_1111_de_2017.pdf

Trabajo,

M. d. (2019,

02

13).

Obtenido

de

https://id.presidencia.gov.co/Documents/190219_Resoluciono312EstandaresMinimo sSeguridadSalud.pdf

Vargas, A. L. (2016, 05 27). lacalera-cundinamarca.gov.co. Obtenido de http://www.lacaleracundinamarca.gov.co/Transparencia/Normatividad/ACUERDO\%20005\%20DE\%202 016\%20PDM\%20OK\%2O(1).pdf

ómica. 